\title{
$\gamma$-Synuclein as a Cancer Biomarker: Viewpoint and New Approaches
}

\author{
Andrei Surguchov $\bowtie$ \\ Department of Neurology, Kansas University Medical Center, Kansas City, 3901 Rainbow Boulevard, Kansas City, KS 66160, USA. \\ $\triangle$ Corresponding author: E-mails: asurguchov@kumc.edu Or apsurguchov@yahoo.com Phone 913-689-0771. \\ (C) Ivyspring International Publisher. This is an open access article distributed under the terms of the Creative Commons Attribution (CC BY-NC) license \\ (https://creativecommons.org/licenses/by-nc/4.0/). See http://ivyspring.com/terms for full terms and conditions.
}

Received: 2016.07.06; Accepted: 2016.07.20; Published: 2016.07.22

\begin{abstract}
Synucleinopathies are complex diseases that progress through several steps, and increasing evidence suggests that an early diagnosis might significantly increase the efficacy of the treatment. A member of the synuclein family, $\alpha$-synuclein is associated with neurodegenerative diseases, whereas another member, $\gamma$-synuclein is implicated predominantly in various forms of cancer. Synucleins can be physiologically secreted to the extracellular space. Accumulating evidence suggests that secretion and cell-to-cell trafficking of both $\alpha$ - and $\gamma$-synuclein may explain the typical patterns of disease progression, however, the molecular mechanisms controlling spreading of pathology are still elusive. Several mechanisms may explain the presence of synucleins in extracellular liquids, including passive diffusion, exosome-mediated secretion, unconventional secretion through exophagy, etc. Analysis of extracellular synucleins in body fluids may reveal the first steps of the disease, become an important diagnostic tool and demonstrate the efficiency of the treatment. $\gamma$-Synuclein is secreted from tumor cells and elevated levels of $\gamma$-synuclein have been detected in various types of cancer, especially in advanced stages of the disease. Urinary analysis of $\gamma$-synuclein is proved to be important for early diagnosis of at least one type of oncologic diseases, bladder cancer. Dynamic intracellular localization of $\gamma$-synuclein and its ability to migrate between cells suggest that this analysis may have diagnostic and prognostic value for other types of cancer.
\end{abstract}

Key words: synucleins, cancer diagnostics, protein secretion, urine biomarkers.

Cancer is a disease that is associated with the progressive accumulation of growth-promoting oncogenes and inactivating changes in tumor-suppressor genes. These variations result in a significant difference in the level of specific protein expression in cancerous cells, which are often reflected in peripheral body fluids. The analysis of biomarkers in body fluids may reveal the first steps of the disease, become an important diagnostic tool, demonstrate the efficiency of the treatment, determine the status of a tumor in vivo, improve patient survival rates, predict clinical outcome etc. Significant progress in cancer diagnostic has been made by analysis of biomarkers in blood, serum, plasma and cerebrospinal fluids. Recently, the importance of urine, containing more than 3,000 detectable protein species became clear for the analysis of cancer biomarkers. Initially urine was used basically for analysis of bladder cancer; however, later studies demonstrated its usefulness for diagnostic and prognosis of other types of tumors, e.g. prostate, kidney and lung cancer [1, 2]. One of the proteins which is elevated in body fluids in various types of cancer and has been first identified because of its 
significant overexpression in breast cancer is $\gamma$-synuclein ( $\gamma$-syn).

$\gamma$-Synuclein ( $\gamma$-syn) is a small naturally unfolded protein belonging to the synuclein family [3]. It was discovered independently by several teams and, therefore, initially received different names: breast cancer specific gene-1 (BCSG1) [4], persyn [5], gamma-synuclein [6] and synoretin [7]. Ultimately the name $\gamma$-synuclein became accepted for convenience. Its association with breast cancer was demonstrated in the very first publication describing $\gamma$-syn by a high-throughput direct-differential cDNA sequencing approach. $\gamma$-Syn was highly expressed in a breast cancer cDNA library, but scarcely in a control breast cDNA library [4]. In situ hybridization demonstrated that $\gamma$-syn is undetectable in normal and benign breast lesions, exhibits partial expression in ductal carcinoma in situ, but is highly expressed in advanced infiltrating breast cancer [4]. Later the association of $\gamma$-syn with many different types of cancer has been demonstrated, including breast, ovarian [8], bladder [9], gastric cancer [10], pancreatic adenocarcinoma [11], glial tumors and medulloblastomas [12]. Furthermore, $\gamma$-syn induces mammary gland proliferation [13], predicts poor clinical outcome in breast cancer [14]. In several studies $\gamma$-syn expression is associated with tumor grade [15]. Elevated levels of $\gamma$-syn proteins have been detected in various types of cancer, especially in advanced stages of the disease. Furthermore, several indicate that overexpression of $\gamma$-syn compromises normal mitotic checkpoint controls, resulting in multinucleation as well as faster cell growth. $\gamma$-Syn has also been shown to promote invasion and metastasis in in vitro assays as well as in animal models [16]. These observations raise questions about the mechanism of involvement of $\gamma$-syn in the process of tumorigenesis and metastasis, and efforts have already been made to use $\gamma$-syn for early cancer detection, as a marker for assessing tumor grade, determination of clinical outcome of cancer progression $[15,16]$.

Finally, it has been established that $\gamma$-syn has a predictive and prognostic value in several types of cancer [17] and may be used as a stage-specific marker in various cancers [18].

$\gamma$-Synuclein can stimulate proliferation and induce invasion and metastasis of breast cancer cells [19]. The clinical follow-up studies confirmed that patients with $\gamma$-synuclein expression predict poor clinical outcome in breast cancer and in colon cancer $[20,21]$.

Investigation of molecular mechanisms underlying the oncogenic functions of $\gamma$-syn has disclosed that its role in cancer may be associated with the membrane-initiated estrogen signaling and related to its chaperone activity [13, 22]. Tissue-specific expression of $\gamma$-syn is regulated on transcriptional level by cis-regulatory elements located in the intron 1 , whereas demethylation of exon 1 is an important factor responsible for the aberrant expression of $\gamma$-syn in cancer $[23,24]$. $\gamma$-Syn expression is also regulated on post-transcriptional level by micro-RNAs [25].

$\gamma$-Syn is secreted from tumor cells and is present in body fluids, e.g. blood, serum, cerebrospinal fluid (CSF), so it was used for diagnostic and prognosis of various types of cancer. In some studies elevated $\gamma$-syn in body fluids, e.g. serum, plasma, blood or CSF, showed diagnostic and/or prognostic properties, however, it did not become a universal cancer marker. Several studies suggest that analysis of $\gamma$-syn in urine may become a convenient method of analysis providing important diagnostic information about several types of cancer. Initially $\gamma$-syn together with two other proteins was identified as a urinary marker for bladder cancer by a combination of proteomic analysis and quantitative western blot [9]. Concomitant use of calreticulin and soluble isoform of catechol-o-methyltransferase together with $\gamma$-syn increased bladder cancer sensitivity detection [9]. Later another team showed that quantitation of $\gamma$-syn expression in bladder tissue correlated with the tumor stage, although $\gamma$-syn tissue level measured by immunohistochemistry was not a prognostic factor for survival prediction [18].

$\gamma$-Syn is also an important urinary diagnostic marker for non-invasive detection of primary and recurrent urothelial urinary bladder carcinoma [26]. The best diagnostic value was achieved when $\gamma$-syn was quantified in combination with another cancer marker - midkine (neurite growth-promoting factor 2, NEGF2) which enhances the angiogenic and proliferative activities of cancer cells [27]. The use of such multi-marker test significantly improves the bladder cancer detection both during the primary diagnostics and subsequent monitoring of patients [26].

Kumar and coauthors (2015) [28] used a high-resolution, mass spectrometry-based, quantitative proteomics approach for measurements variations of potential markers in urine samples from healthy subjects, non-bladder cancer patients and patients with non-invasive and invasive bladder carcinoma [28]. The authors identified $\gamma$-syn and four other robust biomarkers coronin-1A, apolipoprotein A4 and semenogelin-2. The use of such multiplex 
biomarker panel ensures high accuracy for the diagnosis of bladder carcinoma and provides the prospect for a non-invasive way to detect bladder cancer [28].

Liu and coauthors (2016) used immunoassay and western blotting to measure $\gamma$-syn in urine [29] and found that $\gamma$-syn was elevated in the urine of bladder cancer patients. Interestingly, the urine $\gamma$-syn level in patients with urological benign diseases was considerably lower than in bladder cancer patients, but higher than in healthy controls [29].

Thus, urinary analysis of $\gamma$-syn is proved to be important for early diagnosis of at least one type of oncologic diseases, bladder cancer. Dynamic intracellular localization of $\gamma$-syn [30] and its ability to migrate between cells [31] suggest that this analysis may have diagnostic and prognostic value for other types of cancer.

\section{Competing Interests}

The author has declared that no competing interest exists.

\section{References}

1. Husi H, Fearon KC \& Ross JA. Can a simple proteomics urine test assist in the early diagnosis of early-stage cancer? Expert review of proteomics 2011; 8: 555-7.

2. Zhang H, Cao J, Li L, Liu Y, Zhao H, Li N, Li B, Zhang A, Huang H, Chen $\mathrm{S}$, Dong $\mathrm{M}, \mathrm{Yu} \mathrm{L}$, Zhang J, Chen L. Identification of urine protein biomarkers with the potential for early detection of lung cancer. Sci Rep. 2015; 5:11805. doi: 10.1038/srep11805.

3. Surguchov A. Intracellular dynamics of synucleins: Here, there and everywhere. International Review of Cell Molecular Biology, 2015; 320:103-169.

4. Ji H, Liu Y, Jia T, Wang M, Liu J, Xiao G, Joseph B, Rosen C, Shi Y. Identification of a breast cancer-specific gene, BCSG1, by direct differential cDNA sequencing. Cancer Res. 1997; 57: 759-764.

5. Ninkina N, Alimova-Kost M, Paterson J, Delaney L, Cohen B, Imreh S, Gnuchev N, Davies A, Buchman V. Organization, expression and polymorphism of the human persyn gene. Human Mol. Gen. 1998; 7: 1417-1424.

6. Lavedan C, Leroy E, Dehejia A, Buchholtz S, Dutra A, Nussbaum R, Polymeropoulos M. Identification, localization and characterization of the human gamma-synuclein gene. Human Genet. 1998; 103: 106-112.

7. Surguchov A, Surgucheva I, Solessio E, Baehr W. Synoretin--A new protein belonging to the synuclein family. Mol Cell Neurosci. 1999; 13(2):95-103.

8. Nicosia SV, Bai W, Cheng JQ, Coppola D, Kruk PA. Oncogenic pathways implicated in ovarian epithelial cancer. Hematol Oncol Clin North Am. 2003;17 (4):927-43.

9. Iwaki H, Kageyama S, Isono T, Wakabayashi Y, Okada Y, Yoshimura K, Terai A, Arai Y, Iwamura H, Kawakita M, Yoshiki T. Diagnostic potential in bladder cancer of a panel of tumor markers (calreticulin, gamma -synuclein, and catechol-o-methyltransferase) identified by proteomic analysis. Cancer Sci. 2004; 95 (12):955-61.

10. Yanagawa N, Tamura G, Honda T, Endoh M, Nishizuka S, Motoyama T. Demethylation of the synuclein gamma gene $\mathrm{CpG}$ island in primary gastric cancers and gastric cancer cell lines. Clin Cancer Res. 2004; 10 (7):2447-51.

11. Li Z, Sclabas GM, Peng B, Hess KR, Abbruzzese JL, Evans DB, Chiao PJ. Overexpression of synuclein-gamma in pancreatic adenocarcinoma. Cancer. 2004; 101 (1):58-65.

12. Fung KM, Rorke LB, Giasson B, Lee VM, Trojanowski JQ. Expression of alpha-, beta-, and gamma-synuclein in glial tumors and medulloblastomas. Acta Neuropathol. 2003; 106 (2):167-75.
13. Liu YE, Pu W, Jiang Y, Shi D, Dackour R, Shi YE. Chaperoning of estrogen receptor and induction of mammary gland proliferation by neuronal protein synuclein gamma. Oncogene. 2007; 26 (14):2115-25.

14. Guo J, Shou C, Meng L, Jiang B, Dong B, Yao L, Xie Y, Zhang J, Chen Y, Budman DR, Shi YE. Neuronal protein synuclein gamma predicts poor clinical outcome in breast cancer. Int J Cancer. 2007; 121(6): 1296-305.

15. Martin TA, Gomez K, Watkins G, Douglas-Jones A, Mansel RE, Jiang WG. Expression of breast cancer specific gene-1 (BCSG-1/gamma-synuclein) is associated with tumour grade but not with clinical outcome of patients with breast cancer. Oncol Rep. 2006; 16 (1):207-12.

16. Ahmad M, Attoub S, Singh MN, Martin FL, El-Agnaf OM. Gamma-synuclein and the progression of cancer. FASEB J. 2007; 21(13):3419-30.

17. Cirak Y, Furuncuoglu Y, Yapicier O, Alici S, Argon A. Predictive and prognostic values of BubR1 and synuclein-gamma expression in breast cancer. Int J Clin Exp Pathol. 2015; 8 (5):5345-53.

18. Zhao J, Xing N. Identification of gamma-synuclein as a stage-specific marker in bladder cancer by immunohistochemistry. Med Sci Monit, 2014; 20: 2550-2555.

19. Inaba S, Li C, Shi YE, Song DQ, Jiang JD, Liu J. Synuclein gamma inhibits the mitotic checkpoint function and promotes chromosomal instability of breast cancer cells. Breast Cancer Res Treat. 2005; 94(1):25-35.

20. Zou J, Fan YJ, Meng YQ, Xu H, Fan J. An exploratory analysis of $\gamma$-synuclein expression in endometrioid endometrial cancer. BMJ Open. 2012; 2(2): e000611.

21. Liu C, Dong B, Lu A, Qu L, Xing X, Meng L, Wu J, Eric Shi Y, Shou C. Synuclein gamma predicts poor clinical outcome in colon cancer with normal levels of carcinoembryonic antigen. BMC Cancer. 2010; 10:359. doi: 10.1186/1471-2407-10-359.

22. Shi YE, Chen Y, Dackour R, Potters L, Wang S, Ding Q, Wang Z, Liu YE. Synuclein gamma stimulates membrane-initiated estrogen signaling by chaperoning estrogen receptor (ER)-alpha36, a variant of ER-alpha. Am J Pathol. 2010; 177(2):964-73. doi: 10.2353/ajpath.2010.100061

23. Lu A, Gupta A, Li C, Ahlborn TE, Ma Y, Shi EY, Liu J. Molecular mechanisms for aberrant expression of the human breast cancer specific gene 1 in breast cancer cells: control of transcription by DNA methylation and intronic sequences. Oncogene. 2001; 20 (37):5173-85.

24. Gupta A, Godwin AK, Vanderveer L, Lu A, Liu J. Hypomethylation of the synuclein gamma gene $\mathrm{CpG}$ island promotes its aberrant expression in breast carcinoma and ovarian carcinoma. Cancer Res. 2003; 63: 664-73.

25. Surgucheva I, Gunewardena S, Rao HS, Surguchov A. Cell-specific post-transcriptional regulation of $\gamma$-synuclein gene by micro-RNAs. PLOS One, 2013; PONE-D-13- 25172.

26. Soukup V., Kalousová M, Capoun O, Sobotka R, Breyl Z, Pešl M, Zima T, Hanuš T. Panel of Urinary Diagnostic Markers for Non-Invasive Detection of Primary and Recurrent Urothelial Urinary Bladder Carcinoma. Urol Int. 2015; 95: 56-64.

27. Kato M, Shinozawa T, Kato S, Awaya A, Terada T. Increased midkine expression in hepatocellular carcinoma. Arch Pathol Lab Med. 2000; 124 (6):848-52.

28. Kumar P, Nandi S, Tan TZ, Ler SG, Chia KS, Lim WY, Bütow Z, Vordos D, De la Taille A, Al-Haddawi M, Raida M, Beyer B, Ricci E, Colombel M, Chong TW, Chiong E, Soo R, Park MK, Ha HK, Gunaratne J, Thiery JP. Highly sensitive and specific novel biomarkers for the diagnosis of transitional bladder carcinoma. Oncotarget. 2015; 6 (15):13539-49.

29. Liu C, Shi B, Hao C, Wang Q, Lv Q, Xing N, Shou J, Qu L, Gao Y, Qin C, Zhao J, Shou C. Urine gamma-synuclein as a biomarker for the diagnosis of bladder cancer. Oncotarget. 2016; May 19. doi: 10.18632/oncotarget.9468.

30. Surgucheva I, McMahon B, Surguchov A. Dynamic intracellular localization of $\gamma$-synuclein: a clue to its functions. Cell Motility and Cytoskeleton. 2006; 63(8): 447-458.

31. Surgucheva I, Sharov V, Surguchov A. $\gamma$-Synuclein: Seeding of a-Synuclein Aggregation and Transmission Between Cells. Biochemistry. 2012; 51 (23):4743-54. 